\title{
The retarding effect of the TRC response- elimination procedure upon the subsequent reacquisition of autoshaping: Comparison of between- and within-subjects assessment procedures and the evaluation of the role of background contextual stimuli
}

\author{
ARTHUR TOMIE, INES RHOR-STAFFORD, and KATHLEEN T. SCHWAM \\ Rutgers-The State University, New Brunswick, New Jersey 08903
}

\begin{abstract}
In Experiment 1, two groups of pigeons were autoshaped to a green keylight CS and then administered either CS only or truly random control (TRC) response-elimination procedures with the green keylight CS. The groups ceased responding at comparable rates. In a subsequent reacquisition test with a vertical-line key stimulus, the group administered CS only during response elimination reacquired the keypecking $\mathrm{CR}$ more rapidly. The two groups were then administered the alternative response-elimination treatment with the vertical-line CS. Again, the groups ceased responding at comparable rates. In a subsequent reacquisition test with a red keylight CS, the group administered CS only during the immediately preceding response-elimination phase reacquired the keypecking CR more rapidly. In Experiment 2, following initial acquisition, the CS-only and TRC response-elimination treatments were administered under the context-change and no-context change conditions. The TRC/context-change and CS-only/context-change groups ceased responding more rapidly than either the TRC/ no-context-change or CS-only/no-context-change groups. In subsequent reacquisition to a novel $\mathrm{CS}$, the CS-only/no-context-change group reacquired the fastest, the TRC/no-context-change group reacquired the slowest, and the CS-only/context-change and TRC/context-change groups reacquired at similar intermediate rates. Implications of these results for the context-blocking hypothesis are discussed.
\end{abstract}

Although autoshaping is reliably observed when key illumination is followed by the presentation of food, a number of pretraining manipulations have been shown to interfere with the acquisition of the keypecking response. These pretraining manipulations include an uncorrelated relationship between the autoshaping CS and the US (Gamzu \& Williams, 1971, 1973; Mackintosh, 1973; Tomie, Murphy, Fath, \& Jackson, 1980; Wasserman, Franklin, \& Hearst, 1974), an uncorrelated relationship between a stimulus other than the autoshaping CS and the US (Hall \& Honig, 1974; Tomie, 1976a, 1976b, 1980), and intermittent, unsignaled US presentations when no CS is presented (Downing \& Neuringer, 1974; Engberg,

This research was supported by National Institute of Mental Health Grant MH 29425-01, National Science Foundation Grant BNS 77-20564, a Biomedical Sciences Support grant administered by Rutgers University, and Rutgers Research Council grants awarded to the senior author. The authors thank Debi Biehl for her assistance in the analysis of data, and Arthur Murphy, Debi Biehl, and Nick DeFabrizio for their assistance in the running of subjects. Requests for reprints should be sent to Arthur Tomie, Department of Psychology, Busch Campus, Rutgers University, New Brunswick, New Jersey 08903.
Hansen, Welker, \& Thomas, 1972; Tomie et al., 1980; Schwartz \& Balsam, Note 1). Note that each of these procedures exposes the subject to unpredictable presentations of the US.

More recently, Tomie, Hayden, and Biehl (1980) have reported similar effects in a reacquisition test of autoshaping. Following initial acquisition, three groups of pigeons were "extinguished" by different response-elimination procedures. In a subsequent reacquisition test with a novel CS, they noted that groups extinguished with the US present (truly random control; TRC) were retarded relative to a group administered CS only during response elimination. Furthermore, the magnitude of the retardation was observed to be directly related to the amount of experience with unpredictable food which immediately preceded reacquisition testing. It appears, therefore, that reacquisition, as well as the original acquisition of autoshaping, is retarded by extended prior experience with unpredictable food. In all of the previous studies, retardation effects have been evaluated in a between-groups design. The purpose of Experiment 1 was to determine whether similar effects are observed in within-subjects comparisons. 


\section{EXPERIMENT 1}

\section{Method}

Subjects. The subjects were 16 experimental naive adult pigeons obtained from a local supplier and maintained at $75 \%$ of their ad-lib weights throughout the experiment. The subjects were housed in individual metal cages and given free access to grit and water. A 12-h light-dark cycle was in effect in the colony room.

Apparatus. Four standard pigeon chambers were used, with associated automatic programming and recording equipment. The chamber measured $35 \times 35 \times 30 \mathrm{~cm}$ high with a metal intelligence panel containing a $2.9-\mathrm{cm}$-diam pecking key centered $20 \mathrm{~cm}$ above a wire grid floor. Stimuli could be projected onto the response key by Industrial Electronics Engineers in-line display cells equipped with GE 1815 miniature lamps and Kodak Wratten filters 99 and 72B, which provided chromatic light with peak wave-length transmission at 555 and $606 \mathrm{~nm}$, respectively. The display cells also projected a vertical white line which bisected the otherwise unlit response key. The food hopper aperture was located directly below the pecking key. A houselight was mounted behind a 2.4-cm-wide strip of white Plexiglas located above the intelligence panel to provide ambient illumination to the conditioning chamber. Exhaust fans mounted behind the intelligence panel provided air circulation. Eighty-seven decibels (SPL) of masking noise was provided by a Grason-Stadler white-noise generator, Model 1724 .

Procedures. Prior to the initiation of the experiment, the 16 pigeons were unsystematically divided into two groups of eight pigeons each. On Day 1, all pigeons were trained to approach and eat from the food hopper. On Day 2 , all pigeons received the first of 12 daily original acquisition sessions of 60 autoshaping trials per session. Autoshaping trials were administered in a standard Pavlovian delay conditioning procedure, with a 6-sec green $(555 \mathrm{~nm})$ key-light CS and a 4-sec food-hopper US. Trials were programmed according to a variable time (VT) 45-sec schedule. The mean number of keypeck responses per trial over the final 4 days of original acquisition was obtained for each pigeon and used to create two groups matched on asymptotic levels of responding.

On the day following the final original acquisition session, the groups received the first of five daily response-elimination (Resp Elim I) sessions. For both groups, trials were programmed according to the same VT $45-\mathrm{sec}$ schedule used in original acquisition. The CS-only/TRC group were given 60 nonreinforced CS presentations per session, and at the end of each session, were given in their home cages the amount of grain equivalent to 60 US presentations. The TRC/CS-only groups received similar training, except that 60 US presentations were programmed to occur randomly with respect to the CS during each session.

On the day following the final response-elimination session, both groups received the first of eight daily reacquisition (Reacq I) sessions. The procedures were the same as those used in the original acquisition, except that the CS now consisted of a vertical white line which bisected the otherwise dark response key. Immediately following the final session of Reacq I, a second responseelimination phase (Resp Elim II) was administered. This phase consisted of five daily sessions of response elimination with the vertical-line CS comparable to the first response-elimination phase. The TRC/CS-only groups were given 60 nonreinforced CS presentations per session and, at the end of each session, were given in their home cages the amount of grain equivalent to 60 US presentations. The CS-only/TRC group received similar training, except that 60 US presentations were programmed to occur randomly with respect to the CS during each session. Resp Elim II provides, therefore, each group with the extinction treatment administered to the alternative group during Resp Elim I.

On the day following the fifth Resp Elim II session, both groups received the first of eight daily reacquisition (Reacq II) sessions. The procedures were the same as those used in the first reacquisition phase, except that the CS was a red $(606 \mathrm{~nm})$ keylight.

\section{Results}

The mean extinction functions for the first session of Resp Elim I are presented in Figure 1. As shown by the figure, the two groups ceased responding at comparable rates and attained comparable levels of extinction at the end of the session. A two-way mixeddesign analysis of variance, with treatment (CS only vs. TRC) and blocks of five trials as factors, yielded neither a main effect of treatment $[\mathrm{F}(1,14)=2.06$, $\mathrm{p}>.05]$ nor a reliable Treatment by Blocks interaction $[F(11,154)=1.63, p>.05]$. Mean response levels decreased during the remaining four sessions of Resp Elim I for both groups of subjects, and comparable levels of responding were observed for the two groups during Session 5 of Resp Elim I. This observation was confirmed by a two-way mixed-design analysis of variance on the data from Session 5 , which revealed no reliable main effect of treatment $[\mathrm{F}(1,14)=1.25, \mathrm{p}>.05]$ and no Treatment by Blocks interaction $[F(11,154)=1.29, p>.05]$, indicating that the groups entered the Reacq I phase free from any confounding differences in levels of responding.

The mean reacquisition functions for the first two sessions of Reacq I are presented in Figure 1. As revealed by the figure, reacquisition was more rapid following the CS-only treatment than following the TRC treatment, although the groups attained comparable asymptotes. The data from the first two sessions of Reacq I were subjected to the same type of analysis performed on the response-elimination data. A two-way mixed-design analysis of variance with treatment and trials as factors revealed no main effect of treatment $(F<1)$. However, the Treatment by Trials interaction effect was reliable $[F(23,322)$ $=2.31, \mathrm{p}<.01]$, indicating that the groups reacquired at different rates.

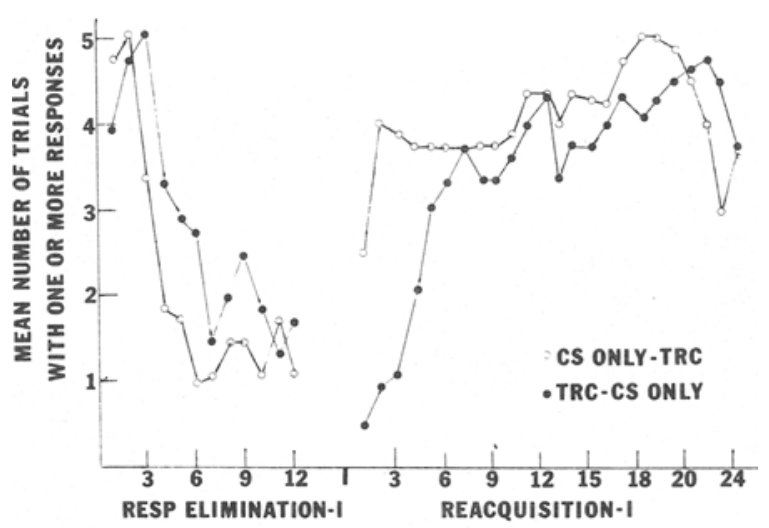

BLOCKS OF FIVE TRIALS

Figure 1. Mean number of trials with one or more responses as a function of five-trial blocks during Session 1 of Resp Elim I and Sessions 1 and 2 of Reacq I for the CS-only/TRC and TRC/CSonly groups. 
The mean extinction functions for the first session of Resp Elim II are presented in Figure 2. As the figure reveals, there were group differences in response levels during the first five trials of the session. The individual data indicated that four of the eight pigeons in the TRC/CS-only group failed to respond during the first block of trials, whereas all of the pigeons in the other group responded on at least two of the first five trials; therefore, it seems that such differences can hardly be attributed to the effectiveness of the treatment. On Blocks 3-12, this effect is reversed and the group administered the CS-only procedure responded somewhat more persistently.

The data from the first response-elimination session were entered into a two-way mixed-design analysis of variance with treatment (CS only vs. TRC) and blocks of five trials as factors. The analysis revealed no main effect of treatment $[F(1,14)=2.36$, $\mathrm{p}>.05$ ] but yielded a reliable Treatment by Blocks interaction $[F(11,154)=2.35, p<.05]$, indicating that the forms of the extinction functions differ reliably. A similar type of analysis was performed on the data from Day 5 of Resp Elim II. That analysis revealed neither a main effect of treatment nor a Treatment by Trials interaction, indicating that the groups entered the Reacq II phase free of confounding differences in levels of responding.

The mean reacquisition function for the first two sessions of Reacq II are presented in Figure 2. As the figure reveals, following response elimination by the CS-only treatment reacquisition is rapid, whereas following response elimination by the TRC treatment reacquisition is profoundly retarded for the first 12 blocks of trials. The two groups attained comparable asymptotes of responding during the remaining seven sessions of Reacq II. The data from the first two sessions of Reacq II were entered into a two-way mixed-design analysis of variance with treatment (CS only vs. TRC) and blocks of five trials as factors.

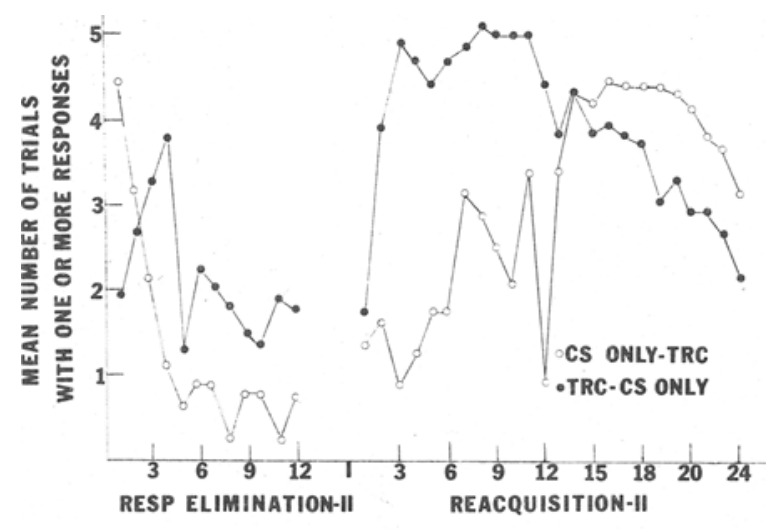

BLOCKS OF FIVE TRIALS

Figure 2. Mean number of trials with one or more responses as a function of five-trial blocks during Session 1 of Resp Elim II and Sessions 1 and 2 of Reacq 11 for the CS-only/TRC and TRC/CS-only groups.
The analysis revealed no main effect of treatment $[F(1,14)=1.67, p>.05]$ but yielded a reliable Treatment by Blocks interaction $[F(23,322)=6.16, p>.01]$, indicating that the forms of the reacquisition functions differ reliably.

In summary, the between-subjects analyses indicate that response elimination was not systematically influenced by the type of procedure employed (CS only or TRC); furthermore, comparable levels of responding were observed during the first and second response elimination phases. Reacquisition, however, was consistently retarded following response elimination by TRC as compared with response elimination by CS only, even though, overall, comparable levels of responding were observed during the first and second reacquisition phases.

This entire constellation of results is supported by the analysis of treatment effects within subjects. No within-subjects effects of response-elimination treatment upon extinction of responding were observed; however, a repeated-tests, mixed-design analysis of variance with groups (CS only-TRC vs. TRC-CS only), tests (Reacq I vs. Reacq II) and blocks of five trials as factors revealed no main effects of groups $[F(1,14)=1.27, p>.05]$ and no main effect of tests $(F<1)$, but did yield a reliable two-way Groups by Tests interaction $[F(1,14)=17.05, p<.01]$ and a reliable three-way Groups by Tests by Blocks interaction $[F(11,154)=3.25, p<.01]$, suggesting that rate of reacquisition for the two groups differed as a function of the response-elimination procedures that preceded it.

In order to ascertain whether the effect of tests was present in both of the groups, separate repeatedtest analyses of variance were performed for the TRC/ CS-only and CS-only/TRC groups. In both cases, the analyses revealed a reliable main effect of test $[F(1,7)$ $=10.42, \mathrm{p}<.01$, and $F(1,7)=7.53, \mathrm{p}<.05$, respectively], indicating that for both groups of pigeons reacquisition was faster following CS-only training than following TRC training.

Inspection of the individual reacquisition functions for the first session of Reacq I and Reacq II for each of the eight pigeons in the TRC/CS-only and CS-only TRC groups reveals that seven of the eight pigeons in the TRC/CS-only group reacquired faster following the CS-only training than following the TRC training. The remaining subject (Subject 6) reacquired very rapidly following both types of pretraining. A similar pattern of results is present in the individual reacquisition functions for the CS-only/TRC group, although the data are somewhat less orderly. For the CS-only/TRC group, Subjects 1 through 4 reacquired faster following the CS-only training than following the TRC training. Subjects 5,6 , and 8 reacquired at similar rates following CS-only and TRC training; however, Subjects 5 and 6 exhibited breakdowns in automaintenance following the initiation of responding during Reacq II. Subject 7 initiated key- 
pecking more rapidly following CS-only training; however, reacquisition performance was erratic in both of the tests.

In summary, within-subjects analysis of reacquisition of autoshaping following CS-only and TRC training revealed that reacquisition was generally more rapid following the former treatment than following the latter, and in no case did a subject reacquire substantially more rapidly following TRC than following CS-only training. These within-subjects effects do not depend upon the sequence in which these procedures were administered, although the effects are more robust when the TRC treatment was given before the CS-only treatment.

\section{Discussion}

The results of this experiment support the following conclusions: (1) Rate of response elimination is not consistently influenced by either type of responseelimination treatment (CS only vs. TRC) or by the presence of previous response-extinction experience (Resp Elim I vs. Resp Elim II) in either betweensubjects or within-subjects comparisons. (2) Reacquisition is retarded by the immediately prior administration of the TRC response-elimination procedure relative to the CS-only procedure. This effect is present in both reacquisition tests as well as in all between-subjects and within-subjects analyses.

Why should response-elimination procedures influence reacquisition but not extinction of the CR? Although no effects were observed in the present experiment, the literature indicates that elimination of Pavlovian CRs is often affected by the presence of the US during extinction (e.g., TRC, explicitly unpaired, backward conditioning); however, the direction of influence has been conspicuously inconsistent. Ayres and DeCosta (1971) and Frey and Butler (1977) have found retardation of response elimination in treatments that retain the US, relative to CS only (cf. Spence, 1966); however, other experimenters (cf. Ayres, Mahoney, Proulx, \& Benedict, 1976; Tomie, Hayden, \& Biehl, 1980, Experiment 1) have noted facilitation of response elimination by procedures retaining the US relative to a CS-only extinction procedure. Finally, as was observed in the present experiment, Tomie, Hayder, and Biehl (1980, Experiment 2) and Rashotte, Griffin, and Sisk (1977) reported the absence of differences in the effectiveness of the TRC and CS-only response-elimination procedures in eliminating the autoshaping $\mathrm{CR}$ in pigeons.

The source of this inconsistency may be inherent in the TRC procedures themselves. Ayres, Benedict, and Witcher (1975) have controlled and monitored the number and relative location of CS-US pairings and have found these factors to be crucial determinants of the effectiveness of the TRC procedure in eliminating the CER in rats. Chance CS-US pairings that occur early during the administration of the TRC procedure apparently condition the CS and thereby interfere with response elimination, while early unpaired USs reduce the effectiveness of later chance CS-US pairings in maintaining the CR. The effects of TRC upon subsequent reacquisition are more consistent, perhaps because reacquisition is evaluated following extended exposure to the TRC procedure.

The retardation of reacquisition following response elimination by TRC relative to CS only is consistent with the Pavlovian and autoshaping literatures. Previous Pavlovian investigators have reported that reacquisition of both conditioned suppression in rats (cf. Ayres \& Decosta, 1971; Ayres et al., 1976) and nictitating membrane conditioning in rabbits (Frey \& Butler, 1977) was retarded by the administration of response elimination procedures which retain the US (e.g., backward conditioning, TRC, explicitly unpaired) relative to CS only. In each of these experiments, the CS used during the reacquisition test was the same as that used during the response-elimination phase.

Recent autoshaping experiments extend this pattern of results to reacquisition testing with a novel CS. Tomie, Hayden, and Biehl (1980) have shown that response elimination by $\mathrm{TRC}$ retards reacquisition to a novel $C S$ relative to response elimination by $C S$ only; furthermore, the magnitude of the retardation effect was shown to be a direct function of the amount of experience with unpredictable food during the response-elimination phase. The results of both Reacq 1 and Reacq II of the present experiment replicate the effects reported by Tomie, Hayden, and Biehl (1980) and extend their observations to withinsubjects comparisons.

The replication of the reacquisition effect withinsubjects indicates that the retarding influence of TRC is reversible and that there are no discernible carryover effect of either response-elimination treatment or prior reacquisition testing on Reacq II performance. Although fewer subjects in the CS-only/TRC group exhibit within-subjects treatment effects, when such effects are observed in this group they tend to be more impressive; consequently, the mean reacquisition functions of the two tests are comparable.

These results are consistent with the context-blocking interpretation advocated by Tomie, Hayden, and Biehl (1980). According to the context-blocking hypothesis, which has been used to account for a number of different types of retardation effects in autoshaping (cf. Tomie, 1976a), the TRC and CS-only procedures provide for conditioning and extinction of the excitatory properties of the context, respectively. The context, therefore, should subsequently exert a stronger blocking influence (when compounded with the illuminated-key CS during reacquisition testing) following the response elimination procedures of the TRC treatment rather than following those of the CS-only treatment. The absence of carry-over effects and the re- 
versibility of retardation observed in the within-subjects design are also consistent with the context-blocking hypothesis, since the hypothesis predicts that withinsubjects variations in the excitatory strength of the context will be systematically related to the magnitude of the blocking effect.

\section{EXPERIMENT 2}

The first experiment replicated the observation that response elimination by TRC subsequently retards the reacquisition of autoshaping relative to response elimination by CS only; furthermore, the effect was observed within subjects as well as between subjects.

The effects of unpredictable food on reacquisition are similar to its effects upon original acquisition. There is considerable evidence that the latter is attributable to the blocking influence of the context, which has become excitatory through pairings with unsignaled food. Retardation, then, should be observed only in an excitatory context. In fact, Tomie (1976a, Experiment 2) has shown that interpolation of nonreinforced context exposure between pretraining and testing attenuates the retardation effect. Since presentation of the context in the absence of food should extinguish its excitatory properties, the results indicate that the effects of context are mediated by the strength of the context-food association. This conclusion is consistent with the context-blocking hypothesis.

Most of the empirical support for the contextblocking hypothesis is derived from experiments that utilized context-shift designs. For example, in one such experiment (Tomie, 1976a), two groups of pigeons received extensive pretraining consisting of random presentations of a red-key CS and food US. the nocontext-change group received this pretraining in the presence of the contextual stimuli that would be present during the acquisition test, while the contextchange group received pretraining in a different context. When both groups were subsequently tested for acquisition to a green-key CS, the no-context-change group was retarded, but the context-change group was not.

The TRC and CS-only response-elimination procedures provide for the presence-or absence-of the US during response elimination which should condition-or extinguish-the context. Therefore, these reacquisition effects may be taken to be analogous to those observed in testing for original acquisition. The retardation of initial acquisition following pretraining with unpredictable food is a context-specific phenomenon. The present experiment examines the contextual specificity of these reacquisition effects in autoshaping.

\footnotetext{
Method

Subjects. The subjects were 40 experimentally naive adult pigeons housed and maintained as in Experiment 1.
}

Apparatus. The apparatus was the same as in Experiment 1. In addition, brown Masonite liners were used. The liners were built to fit the full inside dimensions of the conditioning chamber. Apertures were cut to accommodate the response key and food hopper. The liners included a replaceable brown cardboard floor which covered the wire grid floor of the unlined chamber. The chambers were unilluminated when the liners were in use.

Procedures. Prior to the initiation of the experiment, the 40 pigeons were unsystematically divided into four groups of 10 pigeons each. On Day 1, all pigeons were trained to approach and eat from the food hopper. On Day 2, all pigeons received the first of 12 daily original acquisition sessions of 60 autoshaping trials per session. Autoshaping trials were administered in a standard Pavlovian delay conditioning procedure, with a 6-sec green $(555 \mathrm{~nm})$ keylight CS and a 4-sec food-hopper US. Trials were programmed according to a variable time (VT) $30-\mathrm{sec}$ schedule. The trialsto-criterion scores (number of trials required to attain a criterion of responding for five consecutive trials) and the number of trials with one or more responses for Day 12 were utilized to create four groups of subjects matched for rate of acquisition and asymptotic levels of responding.

On the day following the final pretraining session, the groups were administered the first of five daily response-elimination sessions. The CS-only/no-context-change group was given 60 nonreinforced CS presentations per session programmed according to the same VT 30-sec schedule used in pretraining. The TRC/nocontext-change group was administered similar pretraining, except that 60 US presentations were programmed to occur randomly with respect to the CS. The CS-only/context-change and TRC/ context-change groups received their response elimination procedures in the brown Masonite liners. White noise was used only in conjunction with the use of the unlined chambers.

On the day following the final response-elimination session, the groups received the first of eight daily reacquisition sessions, which were identical to the procedures used in the original acquisition sessions except that the CS now consisted of a vertical white line that bisected the otherwise dark response key.

\section{Results}

The data from the first two sessions of response elimination were entered into a 2 by 2 by 24 mixeddesign analysis of variance with response-elimination treatment (CS only vs. TRC), context (context change vs. no context change), and blocks of five trials as factors. The analysis revealed reliable main effects of treatment $[F(1,36)=18.08, p<.01]$ and context $[F(1,36)$ $=100.75, \mathrm{p}<.01]$, as well as a Treatment by Context interaction $[\mathrm{F}(1,36)=8.31, \mathrm{p}<.01]$. A Fisher's l.s.d. test $(\alpha=.05)$ indicated that the treatment effect in the normal unlined context was less impressive than the treatment effect in the lined context. In the liners, the CS-only group exhibits less responding than the TRC group only in Blocks 3 and 5; in the normal, unlined context, they differ on Blocks $3-9,11-12,14-19$, and 23.

The number of trials to attain a criterion of nonresponding for five consecutive trials was obtained for each subject. The mean trials-to-criterion scores were 5.5, 5.5, 27.9, and 63.7 for the CS-only/contextchange, TRC/context-change, CS-only/no-contextchange, and TRC/no-context-change groups, respectively. The data were entered into a 2 by 2 analysis of variance with treatment and context as factors. The analysis revealed main effects of treatment $[F(1$, 
$36)=7.92, \mathrm{p}<.01]$ and context $[\mathrm{F}(1,36)=40.16$, $\mathrm{p}<.01$ ], as well as a Treatment by Context interaction $[\mathrm{F}(1,36)=7.92, \mathrm{p}<.01]$. A Duncan multiple range test $(\alpha=.05)$ revealed that the CS-only/contextchange and TRC/context-change groups did not differ; however, each of the other groups differed reliably from one another.

The mean response-elimination functions presented in Figure 3 reveal that the CS-only groups ceased responding more rapidly than their TRC counterparts; furthermore, context-change groups extinguished more rapidly than the no-context-change groups. The comparison of the mean response-elimination functions obtained in this experiment with those obtained in Experiment 1 (see Figures 1 and 2) reveals that the slopes and asymptotes of the responseelimination functions of the CS-only groups that did not experience a context change are comparable. The response-elimination function of the TRC/no-contextchange group of this experiment, however, does not resemble the response-elimination functions for either the TRC/CS-only group (see Figure 1) or the CSonly/TRC group (see Figure 2) of Experiment 1. It seems, therefore, that the reliable main effect of treatment and the reliable Treatment by Context interaction observed in the present experiment is attributable to the persistence of responding by subjects in the TRC/no-context-change group and that such persistence is atypical. While there is no immediately obvious explanation for the disparity in performance of the TRC/no-context-change group between Experiments 1 and 2 , it should be noted that the rate of elimination of responding during TRC has been observed to be highly variable (cf. Tomie, Hayden, \& Biehl, 1980).

A 2 by 2 by 12 mixed-design analysis of variance, with treatment, context, and blocks of five trials as

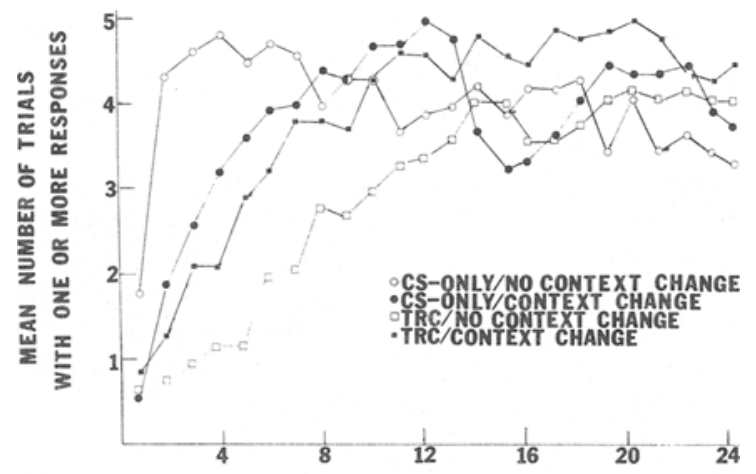

BLOCKS OF FIVE TRIALS

Figure 3. Mean number of trials with one or more responses as a function of five-trial blocks for the CS-only/no-contextchange, CS-only/context-change, TRC/no-context-change, and TRC/context-change groups during the first two sessions of response elimination.

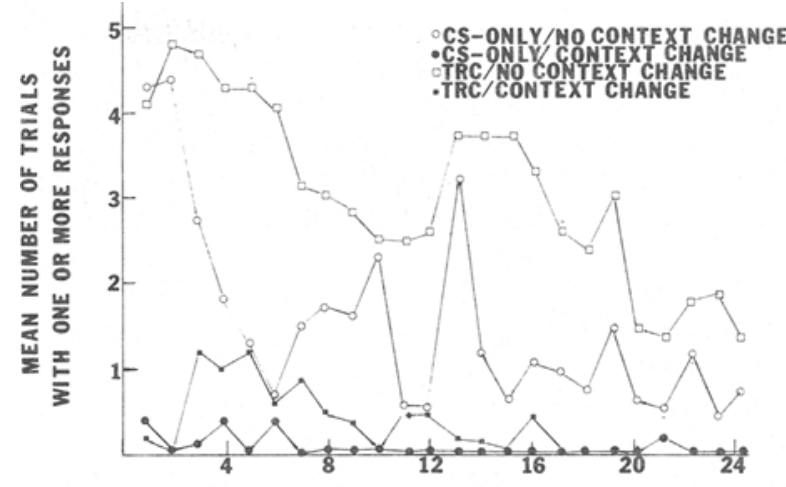

BLOCKS OF FIVE TRIALS

Figure 4. Mean number of trials with one or more responses as a function of five-trial blocks for the CS-only/no-contextchange, CS-only/context-change, TRC/no-context-change, and TRC/context-change groups during the first two sessions of reacquisition.

factors, was performed on the data from Session 5 of response elimination. The analysis revealed no main effects or interaction effects which approached statistical significance, indicating that the groups ceased responding to the same degree and entered the reacquisition test free of confounding differences in level of responding.

The mean reacquisition functions for each of the four groups are presented in Figure 4. As revealed by the figure, the CS-only/no-context-change group reacquired the fastest, the $\mathrm{TRC} /$ no-context-change group reacquired the slowest, and the CS-only/ context-change and TRC/context-change groups reacquired at similar intermediate rates. The data were entered into a 2 by 2 by 24 mixed-design analysis of variance with treatment (CS only vs. TRC), context (context change vs. no context change), and blocks of five trials as factors. The analysis revealed no main effects of treatment $[F(1,36)=1.12, p>.10]$ or context $(F<1)$. However, a reliable Treatment by Blocks interaction was found $[F(23,828)=6.90, p<.01]$, indicating that the reacquisition functions for the CSonly groups were steeper than those for the TRC groups. In addition, the analysis yielded a reliable three-way Treatment by Context by Blocks interaction $[F(23,828)=1.97, p<.01]$, indicating that the treatment effects in the test context were more impressive than the treatment effects out of the text context.

A Fisher's l.s.d. test $(\alpha=.05)$ revealed that the CSonly/no-context-change group reacquired the fastest, differing from CS-only/context change (Blocks 2-4), TRC/context change (Blocks 2-6), and TRC/no context change (Blocks 2-6, 9); the TRC/no-contextchange group was the slowest, differing from TRC/ context change (Blocks 5,7 ) and CS only/context change (Blocks 4-12). The CS-only/context-change and TRC/context-change groups never differed. 
The number of trials to attain a criterion of responding for five consecutive trials was obtained for each subject. The mean trials-to-criterion scores were $11.6,24.2,30.4$, and 54.3 for the CS-only/no-contextchange, CS-only/context-change, TRC/contextchange, and $\mathrm{TRC} /$ no-context-change groups, respectively. The data were entered into a simple twoway analysis of variance with treatment and context as factors. The analysis revealed a main effect of treatment $[F(1,36)=10.36, p<.01]$, no effect of context $(F<1)$, and a reliable Treatment by Context interaction $[F(1,36)=5.77, p<.02]$. A Duncan multiple range test $(\alpha=.05)$ revealed that the TRC/ no-context-change group was reliably retarded relative to the other three groups, which did not differ from each other.

There were no discernible differences in asymptotic levels of responding in Sessions 2 through 8 . A 2 by 2 by 12 mixed-design analysis of variance with treatment, context, and blocks of five trials as factors was performed on the data from Session 8 of reacquisition. The analysis revealed no main effects or interaction effects that approached significance, indicating that the groups reacquired to comparable asymptotes.

Pearson's product moment correlation coefficients were computed for the trials-to-criterion data from initial acquisition, response elimination, and reacquisition. For each subject within a group, the trials-tocriterion scores for all three phases were intercorrelated. None of the correlations approached significance, indicating that the speed of both response elimination and reacquisition was not correlated with the speed of initial acquisition, and also that the speed of reacquisition was not correlated with the speed of response elimination.

\section{Discussion}

The results of this experiment indicate that the extinction of responding is retarded by the TRC responseelimination procedure; however, this result is atypical (i.e., not observed in either Resp Elim I or II of Experiment 2 or in previous autoshaping studies) and is probably representative of the noted variability often observed in such TRC procedures. The most striking aspect of the response-elimination data is the absence of responding by subjects in the contextchange groups. The absence of keypecking during response elimination by the majority of the subjects in the context-change groups was not completely unexpected. Wasserman (1973) and Tomie (1976a) have reported little autoshaping in an unilluminated context. The present results extend the constraints of contextual illumination upon autoshaping to the maintenance of a previously acquired response and might indicate that the context was an essential part of the stimulus complex that controls behavior. Observation of the pigeons in the lined chambers reveals that they were virtually stationary except when feeding from the magazine. Their normal patterns of stereotyped adjunctive behaviors (cf. Staddon \& Simmelhag, 1971) that typically accompany the autoshaping response were conspicuously absent. The notion that the keypecking CR is a terminal component of a more elaborate adjunctive sequence seems well supported by these observations, and suggests that the absence of keypecking may be a direct. effect of the absence of illumination, rather than an associative contextual consideration.

There is at least one associative explanation of the abrupt cessation of responding by subjects in the context-change groups. If one assumes that $\mathrm{CR}$ strength is directly related to the associative value of all CSs present on the trial (i.e., CS-context summation hypothesis), then the absence of responding following the change in context is attributable to the low associative strength of the novel context. While the CS-context summation hypothesis is consistent with the entire constellation of response elimination results observed in Experiment 2, it does not predict the reacquisition effects of either the responseelimination treatment factor or the context factor.

The reacquisition results of this experiment are consistent with the results of both reacquisition phases of Experiment 1. Moreover, they allow for more precise specification of the nature of the treatment effects. For example, the reacquisition differences following CS-only vs. TRC treatments that were observed in Experiment 1 may be due to the facilitation of reacquisition following $C S$ only, the retardation of reacquisition following TRC, or a combination of both. According to the context blocking hypothesis, the CS-only procedure extinguishes the excitatory properties of the context while the TRC procedure does precisely the opposite. The context-shift design utilized in Experiment 2 allows for assessment of a baseline rate of reacquisition, against which effects of treatment could be evaluated since context change groups were not exposed to either extinction or conditioning of the test context. The results presented in Figure 4 reveal that the CS-only/ no-context-change group reacquired faster than the context-change groups, while the TRC/no-contextchange group reacquired more slowly than the contextchange groups. It appears, therefore, that the CS-only and TRC treatments facilitate and retard reacquisition, respectively, and that their influences are context-specific. This entire constellation of results is consistent with the context-blocking hypothesis.

Context-induced nonresponding during response elimination does not appear to directly influence reacquisition performance. In the present experiment, two groups, TRC/context change and CS only/context change, failed to respond during response elimination, and, while both groups were facilitated in reacquisition relative to the $\mathrm{TRC} /$ no-context-change group, neither group reacquired as rapidly as the CSonly/no-context group. If the effects of treatment 
upon reacquisition are attributable to the presence or absence of responding during response elimination, then the context-change factor should exercise a consistent effect across treatments. The reacquisition results of Experiment 2 are clearly inconsistent with such an analysis.

At this point, it should be noted that in these experiments, as in related research (Tomie, 1976a, 1976b; Tomie, Hayden, \& Biehl, 1980), the test for the (re)acquisition of autoshaping was conducted only in the normal unlined chamber. This confounds the presence or absence of a context change with the locus of the treatment, and the data, consequently, are subject to interpretation along those lines. Appropriate counterbalancing would require the administration of autoshaping in the unilluminated lined chamber, where autoshaping is typically not observed. The alleviation of this confound by manipulations of the auditory context which does not directly affect the acquisition of autoshaping (Tomie, 1976a, Experiment 3) reveals that effects such as those reported here are due to the presence or absence of a context change rather than to the specific locus of the treatment.

\section{GENERAL DISCUSSION}

The results of these experiments support the following conclusions: (1) There are no consistent differences in rates of response elimination produced by the CS-only and TRC treatments. In Experiment 1, the two procedures produced similar rates of extinction. In Experiment 2, the TRC/no-contextchange group maintained a higher level of responding than did the CS-only/no-context-change group; however, the response-elimination function obtained from the former group was atypical. (2) The contextchange groups, irrespective of treatment, are marked by the immediate suppression of responding. The majority of the subjects in each of the context-change groups did not keypeck at any time during the response-elimination phase. Subjects in the nocontext change groups, on the other hand, show the gradual diminution of response strength typically observed in such extinction procedures. (3) When response-elimination treatments are administered in the context, the CS-only groups reacquire to a novel CS more rapidly than do the TRC groups (Experiments 1 and 2); moreover, these effects are observed within subjects (Experiment 1). However, when response-elimination treatments are administered out of the text context (in the liners), such treatment effects are not observed (Experiment 2). (4) The nocontext-change groups are either facilitated or retarded in reacquisition relative to the context-change groups, depending upon the response-elimination treatment administered. The CS-only/no-contextchange group is facilitated relative to the CS-only/ context-change group (Experiment 2), while the
$\mathrm{TRC} /$ no-context-change group is retarded relative to the TRC/context-change group (Experiment 2).

The congruence of this constellation of results with the context-blocking hypothesis warrants further inspection. It appears that the hypothesis readily accounts for the reacquisition data. Context blocking would predict [see (3) above] the in-context treatment effect as well as the absence of such an effect in the liner condition. Context blocking also predicts [see (4) above] that treatments which provide for context extinction (e.g., CS only) will facilitate reacquisition in context, whereas treatments which provide for context-US pairings (e.g., TRC) will retard reacquisition in context. Such interpretations have been applied to retardation effects in the initial acquisition in autoshaping (cf. Engberg et al., 1972; Hall \& Honig, 1974; Mackintosh, 1973).

The reacquisition data are consistent with the context-blocking hypothesis; however, the evaluation of the data with respect to alternative theoretical accounts is appropriate. It is possible to view the retarded reaquisition of autoshaping as a general transfer of training effect that is a by-product of the associative impairment engendered by TRC training wherein the US is unpredictable. Their application to these reacquisition effects, however, seems less appropriate, since all subjects have common history of previous autoshaping during initial acquisition which should provide all subjects with common cognitions (i.e., key illumination predicts food) as well as relatively specific "immunization" against the deleterious effects of cognitive associative interference. The transfer of training accounts, moreover, predict neither the facilitation of reacquisition engendered by CS-only training nor the contextual specificity of the facilitation and retardation effects. The withinsubject effects observed in Experiment 1 are particularly difficult to accommodate within a cognitive transfer-of-training framework. The reversibility of reacquisition performance by the alteration in the response elimination procedures indicates that the mediating cognition learned during TRC is either ephemeral or frail, or both.

An alternative interpretation of retardation in autoshaping suggests that unpredictable US presentations establish competing behaviors that are superstitiously reinforced by instrumental acquisition processes (Gamzu, Williams, \& Schwartz, 1973). These behaviors are, ostensibly, incompatible with the autoshaping response and thereby interfere with the acquisition of keypecking. There are several problems with the competing-response interpretation. Behaviors that develop as a direct consequence of intermittent US presentations are not incompatible with the topography of the autoshaping response, but, rather, would tend to bring the pigeon into the vicinity of the pecking key and promote orientation toward that stimulus (Staddon \& Simmelhag, 1971). The assumption that instrumentally maintained 
motor responses necessarily retard the acquisition of autoshaping is also highly questionable. LoLordo, McMillan, and Riley (1974) observed that pigeons would readily autoshape while concomitantly maintaining a well-learned treadle-press response. Engberg et al. (1972) explicitly trained an incompatible response (treadle-pressing) for 30 days prior to the initiation of autoshaping. Such training facilitated rather than retarded the acquisition of keypecking.

The data reported here are particularly difficult to accommodate within a competing-response framework. To the degree that superstitious acquisition is a factor, one would expect that during initial acquisition, behaviors compatible with keypecking would be temporally contiguous with food and hence instrumentally strengthened. Responses compatible with keypecking would therefore be most likely to be maintained during the response-elimination phase by the TRC treatment. The maintenance of such behaviors should serve to facilitate rather than retard the reacquisition of keypecking. Of further embarrassment to the competing-response position, the context manipulation, which is observed to elicit behavior highly incompatible with keypecking (freezing), fails to retard reacquisition following the TRC treatment.

In summary, it appears that the reacquisition of autoshaping is retarded by the administration of the TRC procedure and facilitated by the administration of the CS-only procedure and that these treatment effects are context-specific and are observed within subjects. The reacquisition results are consistent with the context-blocking hypothesis and theories of Pavlovian conditioning, which emphasize the role of contextual stimuli (Gibbon \& Balsam, in press; Rescorla \& Wagner, 1972) and suggest that manipulations be included to control for contextual influences in assessments of reacquisition following differential response elimination.

\section{REFERENCE NOTE}

1. Schwartz, A. L., \& Balsam, P. D. Retardation of autoshaping following US-only pretraining. Paper presented at the meeting of the Eastern Psychological Association, Philadelphia, April 1979.

\section{REFERENCES}

Ayres, J. J. B., Benedict, J. C., \& Witcher, E. S. Systematic manipulation of individual events in a TRC procedure in rats. Journal of Comparative and Physiological Psychology, 1975. 88, 97-103.

Ayres, J. J. B., \& DeCosta, M. J. The TRC as an extinction procedure. Psychonomic Science, 1971, 24, 31-33.

Ayres, J. J. B., Mahoney, W. J., Prouly, D. T., \& Benedict, J. L. Backward conditioning as an extinction procedure. Learning and Motivation, 1976, 7, 368-381.

Downing, K., \& Neuringer, A. Autoshaping as a function of prior food presentations. Journal of the Experimental Analysis of Behavior, 1976, 26, 465-469.
Engberg, L. A., Hansen, G., Welker, R. L., \& Thomas, D. R. Acquisition of key-pecking via autoshaping as a function of prior experience: "Learned laziness?" Science, 1972, 178, 10021004.

Frey, P. W., \& Butler, C. S. Extinction after aversive conditioning: An associative or nonassociative process? Learning and Motivation, 1977, 8, 1-17.

Gamzu, E., \& Williams, D. R. Classical conditioning of a complex skeletal response. Science, 1971, 171, 923-925.

Gamzu, E., \& Williams, D. R. Associative factors underlying the pigeon's keypecking in autoshaping procedures. Journal of the Experimental Analysis of Behavior, 1973, 19, 225-232.

Gamzu, E., Williams, D. R., \& Schwartz, B. Pitfalls of organismic concepts: "Learned laziness?" Science, 1973, 181, 367-368.

Gibbon, J., \& Balsam, P. D. Spreading association in time. In C. M. Locurto, H. S. Terrace, \& J. Gibbon (Eds.), Autoshaping and conditioning theory. New York: Academic Press, in press.

Hall, G., \& Honig, W. K. Stimulus control after extradimensional training in pigeons: A comparison of response contingent and noncontingent training procedures. Journal of Comparative and Physiological Psychology, 1974, 87, 945-952.

LoLordo, V. M., McMillan, J. C., \& Riley, A. L. The effects upon food-reinforced pecking and treadle-pressing of auditory and visual signals for response-independent food. Learning and Motivation, 1974, 5, 24-41.

Mackintosh, N. J. Stimulus selection: Learning to ignore stimuli that predict no change in reinforcement. In R. A. Hinde \& J. Stevenson-Hinde (Eds.), Constraints on learning. New York: Academic Press, 1973.

Rashotte, M. E., Griffin, R. W., \& Sisk, C. L. Second-order conditioning of the pigeon's keypeck. Animal Learning \& Behavior, 1977, 5, 25-38.

Rescorla, R. A., \& Wagner, A. R. A theory of Pavlovian conditioning: Variations in the effectiveness of reinforcement and nonreinforcement. In A. H. Black \& W. F. Prokasy (Eds.), Classical conditioning II: Current theory and research. New York: Appleton-Century-Crofts, 1972.

Spence, K. W. Cognitive and drive factors in the extinction of the conditioned eyeblink in human subjects. Psychological Review, 1966, 73, 445-458.

Staddon, J. E. R., \& Simmelhag, V. L. The "superstition" experiment: A re-evaluation of its implications for the principles of adaptive behavior. Psychological Review, 1970, 78, 3-43.

Tomis, A. Interference with autoshaping by prior context conditioning. Journal of Experimental Psychology: Animal Behavior Processes, 1976, 2, 323-334. (a)

Tomie, A. Retardation of autoshaping: Control by contextual stimuli. Science, 1976, 192, 1244-1246. (b)

Tоміе, A. Effects of unpredictable food upon the subsequent acquisition of autoshaping: Analysis of the context blocking hypothesis. In C. M. Locurto, H. S. Terrace, \& J. Gibbon (Eds.), Autoshaping and conditioning theory. New York: Academic Press, in press.

Tomie, A., Hayden, M., \& Biehl, D. Effects of response elimination procedures upon the subsequent reacquisition of autoshaping. Animal Learning \& Behavior, 1980, 8, 237-244.

Tomie, A., Murphy, A. L., Fath, S., \& Jackson, R. L. Retardation of autoshaping following pretraining with unpredictable food: Effects of changing the context between pretraining and testing. Learning and Motivation, 1980, in press.

Wasserman, E. A. The effect of redundant contextual stimuli on autoshaping the pigeon's keypeck. Animal Learning \& Behavior, 1973, 1, 198-206.

Wasserman, E. A., Franklin, S. R., \& Hearst, E. Pavlovian appetitive contingencies and approach vs withdrawal to conditioned stimuli in pigeons. Journal of Comparative and Physiological Psychology, 1974, 86, 616-627.

(Received for publication July 2, 1980; revision accepted December 5,1980 .) 